\title{
What we have learned from randomized trials of prostate cancer screening
}

\author{
Richard M Hoffman ${ }^{1,2}$ and Anthony Y Smith ${ }^{3}$
}

The introduction of prostate-specific antigen (PSA) for prostate cancer screening in the late 1980s led to an epidemic of prostate cancer, particularly in developed countries. However, the first valid reports from randomized controlled trials on the efficacy of screening were not published until 2009. Men in the screening group in the European Randomized Study of Screening for Prostate Cancer were $20 \%$ less likely than those in the control group to die from prostate cancer. The absolute difference was only $0.7 / 1000$, implying that over 1400 men needed to be screened to prevent one prostate cancer death. Screening was also associated with a $70 \%$ increased risk for being diagnosed with prostate cancer. The American Prostate, Lung, Colorectal, and Ovarian Cancer Screening Trial found no survival benefit for screening. Results were not conclusive because a substantial proportion of study subjects had previously undergone PSA testing, over half of the control group had PSA testing, follow-up was relatively short, and fewer than 100 subjects died from prostate cancer. Balancing the potential survival benefit from screening is the risk of overdiagnosis-finding cancers that would not otherwise cause clinical problems - and the risk of treatment complications, including urinary, sexual and bowel dysfunction.

Prostate cancer screening efforts would benefit from improved biomarkers, which more readily identify clinically important cancers. Cancer control efforts might also need to include chemoprevention, though currently available agents are controversial. In the meantime, patients need to be supported in achieving informed decisions on whether to be screened for prostate cancer. Asian Journal of Andrology (2011) 13, 369-373; doi:10.1038/aja.2010.181; published online 11 April 2011

Keywords: cancer screening; prostate-specific antigen; prostatic neoplasms; randomized controlled trials as topic

\section{BACKGROUND}

The introduction of the prostate-specific antigen (PSA) assay revolutionized prostate cancer screening. Men with prostate cancer were found to have higher concentrations of PSA, a kallikrein-like serine protease produced by prostatic epithelial cells, than men with normal prostates or benign conditions. The US Food and Drug Administration approved PSA testing in 1986 for cancer monitoring, thought it was also being considered for screening. ${ }^{1}$ After observational studies began reporting that PSA was more sensitive for detecting prostate cancer than digital rectal examination (DRE) and more likely to detect cancer at an early stage, ${ }^{2,3}$ organizations such as the American Cancer Society and the American Urological Association began strongly recommending routine screening for men over age $50 .{ }^{4,5}$ Consequently, PSA testing was quickly and widely adopted for prostate cancer screening in the United States, with the US Food and Drug Administration approving this indication in 1994. ${ }^{1}$ By 2001, the US Behavioral Risk Factor Surveillance System survey estimated that $75 \%$ of American men over age 50 had undergone PSA testing. ${ }^{6}$

PSA screening led to an epidemic of prostate cancer, with the lifetime risk of cancer diagnosis in the United States increasing from 9\% to $16 \%$ and the number of cases being diagnosed each year increasing from around 90000 in $1986^{7}$ to nearly 220000 in $2010 .^{8}$ While most countries were more cautious in implementing PSA screening than the United States, the International Agency for Research on Cancer estimated that in 2008 there were nearly one million cases diagnosed and about 258000 deaths from prostate cancer worldwide. ${ }^{9}$ International Agency for Research on Cancer further reported that prostate cancer was the second most commonly diagnosed cancer among men and the second leading cause of cancer death. Incidence rates varied 25 -fold, with nearly three-quarters of the cases diagnosed in developed countries where PSA testing was more widespread, though these ecologic disparities could have partly been due to reporting biases. However, mortality rates were less variable and were quite similar between more and less developed countries.

An effective screening test should lead to a shift in cancer stage at diagnosis, with a decrease in the incidence of distant stage disease. Such an effect was observed in the United States, though with a quite disproportionate concomitant increase in the incidence of localized cancer. ${ }^{10}$ Cancer mortality rates also decreased in the United States, dropping from an estimated 34.9 per 100000 in 1986 to 23.5 per 100 000 in $2007 .^{8}$ However, the absolute reduction in the lifetime risk of every dying from prostate cancer was only from $3.4 \%$ to $2.8 \%$. Additionally, declining mortality rates cannot necessarily be fully attributed to screening; better treatments, particularly for advancedstage disease, and biases in completing death certificates might also be contributing factors. ${ }^{11}$ A modeling study of cancer epidemiology data in the United States estimated that $45 \%-70 \%$ of the mortality decrease could be attributed to screening. ${ }^{12}$ 


\section{RANDOMIZED CONTROLLED TRIALS OF PROSTATE CANCER} SCREENING

The most convincing evidence that screening is effective comes from randomized controlled trials, where men are randomly assigned to screening or usual care and followed to see whether screening reduces cancer mortality and morbidity. Major screening trials were implemented in the early 1990s, including the European Randomized Study of Screening for Prostate Cancer (ERSPC) ${ }^{13}$ and the American Prostate, Lung, Colorectal, and Ovarian Cancer Screening Trial (PLCO). ${ }^{14}$ These studies, which combined enrolled over 200000 men aged 50-74 years, were expected to take at least a decade before any survival benefit was observed.

In the meantime, a randomized trial from Quebec reported that prostate cancer screening reduced prostate cancer mortality within only 8 years of initiating the study in $1988 .{ }^{15}$ Investigators used electoral rolls to randomize men aged $45-80$ years to be invited for screening ( $n=30956)$ or usual care $(n=15237)$ after excluding men with previous prostate cancer diagnoses or screening. Labrie and colleagues reported a $67 \%$ reduction in cancer mortality, from 41.6 per 100000 person-years to 13.7 per 100000 person-years.

However, the Quebec study results were challenged on numerous levels. Given the often indolent nature of prostate cancer, seeing a survival benefit within such a short time seemed biologically implausible. PSA is estimated to detect prostate cancer 5-10 years before it becomes clinically detectable; ${ }^{16,17}$ treatment trials show that prostate cancer mortality is quite uncommon in the subsequent 4 years following clinical detection even in the untreated group. ${ }^{18}$ More importantly, the Quebec results did not reflect an intention to screen analysis, where all outcomes are attributed based on the original group assignment-either to screening or control. Instead, the investigators excluded subjects assigned to screening who were non-compliant with screening and excluded control subjects who underwent screening. When results were analyzed according to the study assignment, as reported in a Cochrane Collaboration Review, the mortality rates were essentially identical, with a relative risk of 1.01 (95\% confidence inter$\operatorname{val}(\mathrm{CI}): 0.76-1.33) .{ }^{19}$

After the publication of the unconvincing Canadian results, and while ERSPC and PLCO were ongoing, considerable evidence was accruing suggesting that PSA screening could potentially be harmful. One problem was the substantial risk for overdiagnosis-finding cancers that would not otherwise be destined to cause problems during a man's lifetime. Estimates for overdiagnosis, based on modeling data from the National Cancer Institute's Surveillance Epidemiology and End Results registries and Medicare as well as the ERSPC trial, ranged from $23 \%$ to $42 \%{ }^{20}$ Aside from the psychological burden of the cancer diagnosis, overdiagnosis directly leads to overtreatment. Most screening-detected cancers are early-stage and the increased cancer incidence led to marked increase in the use of surgery and radiation. ${ }^{21,22}$ These treatments can cause complications, particularly urinary, sexual and bowel dysfunction. ${ }^{23}$ Radical prostatectomy also has a mortality rate that can reach $1 \%$ in older men. ${ }^{24}$

The recognition that screening can lead to unwanted outcomes eventually led to a consensus among professional society guidelines that men be informed about the risks and benefits of early detection and treatment and allowed to decide whether to be screened. ${ }^{25-27} \mathrm{An}$ underlying theme in all of the guidelines was the expectation that the results of the ERSPC and PLCO would provide substantial guidance for screening recommendations.

Finally, in March 2009, results from the ERSPC and PLCO were published in the New England Journal of Medicine (Table 1). ${ }^{28,29}$ The ERSPC was a multinational study with a complicated study design that used different strategies to enroll subjects and to perform screening. ${ }^{28}$ Subjects were randomized from population-based registries in Finland, Sweden and Italy to be invited for screening or to be in the control arm, while subjects in the Netherlands, Belgium, Switzerland and Spain were consented and then randomized to either screening or control arms. All sites screened with PSA alone at 4-year testing intervals except for Sweden, which used a 2 -year interval. Most centers used a PSA level of $3.0 \mathrm{ng} \mathrm{ml}^{-1}$ as the cutoff for biopsy referral, though some centers variably incorporated PSA velocity, free PSA, DRE, and ultrasound results. Prostate cancers were treated according to local policies and guidelines. Investigators used an algorithm to determined cause of death through blinded assessments of registries and medical records.

The ERSPC enrolled 182160 subjects aged 50-74 years between 1991 and 2003, though the initial report was based on the 162243 men aged 55-69 years. The average age at enrollment was 61.7 years for both the screening and control groups; no data were collected on family history of prostate cancer or previous screening. The subjects were followed for a median of 9 years. Overall, $82.2 \%$ of the screening group had at least one PSA test and $16.2 \%$ of tests were abnormal. The cancer detection rate was $70 \%$ higher in the screening group than in the control group $(8.2 \%$ versus $4.8 \%$, rate ratio $=1.70,95 \%$ CI: 1.64-1.77). The screening group $(n=214)$ had significantly fewer deaths than the control group $(n=326)$ for a rate ratio of $0.80(95 \%$ CI: 0.65-0.98). The screening group also had a significantly lower risk for being diagnosed with clinically advanced tumors $(0.9 / 1000$ personyears versus $1.15 / 1000$ person-years, $P<0.0001$ ).

Although screening was associated with a significant reduction in the risk for dying from prostate cancer, the absolute risk reduction was only $0.7 / 1000$ men, implying that over 1400 men needed to be screened approximately twice over 9 years to prevent one prostate cancer death. Furthermore, the 70\% increased risk for cancer diagnosis in the screening group meant that 48 men needed to be diagnosed for every prostate cancer death prevented, about three-quarters of whom received active treatment. Additionally, the ERSPC subjects received sextant biopsies; given that current recommendations for prostate biopsy are to obtain at least 12 samples, ${ }^{30}$ the higher risk for cancer diagnosis may be an underestimate.

Table 1 Results from the European Randomized Study of Screening for Prostate Cancer (ERSPC) and Prostate, Lung, Colorectal, and Ovarian Cancer Screening Trial (PLCO)

\begin{tabular}{|c|c|c|c|c|c|c|}
\hline \multirow[t]{2}{*}{ Study } & \multirow[t]{2}{*}{ Study group } & \multirow[t]{2}{*}{ Age (years) } & \multicolumn{2}{|c|}{ Prostate cancer detection } & \multicolumn{2}{|c|}{ Prostate cancer deaths } \\
\hline & & & $\mathrm{N}$ & Rate ratio $(95 \% \mathrm{Cl})$ & $\mathrm{N}$ & Rate ratio $(95 \% \mathrm{Cl})$ \\
\hline \multirow[t]{2}{*}{$\mathrm{ERSPC}^{28}$} & Screening $(n=72890)$ & $55-69$ & 5990 & $1.70(1.64-1.77)$ & 214 & $0.80(0.65-0.98)$ \\
\hline & Control $(n=89353)$ & & 4307 & & 326 & \\
\hline \multirow[t]{2}{*}{$\mathrm{PLCO}^{29}$} & Screening $(n=38343)$ & $55-74$ & 2820 & $1.22(1.16-1.29)$ & 50 & $1.13(0.75-1.70)$ \\
\hline & Control $(n=38350)$ & & 2322 & & 44 & \\
\hline
\end{tabular}


Even though screening was being offered far less often in Europe than in the United States, subjects in the ERSPC control group were still being tested with PSA. The initial publication did not report on the extent of contamination, but indicated that $31 \%$ of cancers in the control group were stage T1c, implying that they were diagnosed as a result of PSA testing. Additionally, $23.9 \%$ of men randomized to the screening arm did not attend the initial screening round. A post hoc analysis of the ERSPC data estimated the prostate cancer mortality reduction associated with screening after adjusting for both contamination and nonattendance. ${ }^{31}$ Investigators used national laboratory data and surveys to identify PSA screening in the Rotterdam site controls, and then extrapolated these data to the entire ERSPC cohort. After adjusting for nonattendance and contamination, PSA screening was estimated to reduce prostate cancer mortality by $31 \%$ (95\% CI: $8 \%-49 \%$ ) to $29 \%$ (95\% CI: 7\%-45\%), depending upon the definition of contamination. Another analysis of the Rotterdam site estimated the benefit of PSA screening for reducing the risk of metastatic prostate cancer. ${ }^{32}$ Among the 42376 men aged 55-75 years, unadjusted analyses estimated a $25 \%(95 \%$ CI: $5 \%-41 \%)$ reduction in the occurrence of metastatic prostate cancer. However, the risk reduction increased to 32\% (95\% CI: 6\%-51\%) after adjusting for both contamination and non-compliance.

The Goteborg Sweden site subsequently reported a 44\% (95\% CI: $18 \%-61 \%)$ relative risk reduction in mortality among the cohort of men aged 50-64 years invited for PSA testing compared to uninvited controls after follow-up for a median of 14 years. ${ }^{33}$ Among attendees, the relative risk reduction was $56 \%$ (95\% CI: $32 \%-72 \%$ ) compared to controls. The Goteborg screening group underwent PSA testing every 2 years compared to the every 4 -year interval for the other ERSPC sites. The additional benefit with longer follow-up is plausible. Studies have estimated a 4 - to 10-year lead time with PSA screening, ${ }^{16,20}$ and survival curves following treatment for clinically detected cancers do not diverge for at least 4 years. ${ }^{18}$ Additionally, the Swedish analyses focused only on men aged 50-64 years, a younger cohort than reported in the initial ERPSC publication, and one more likely to see a survival benefit with aggressive treatment. ${ }^{18}$ However, the wide CI included the $20 \%$ benefit reported previously and PSA testing was also associated with a $64 \%$ increase in cancer diagnosis.

The PLCO did not find any survival benefit with screening. The PLCO enrolled 76693 men between the ages of 55 and 74 years from 1993 to $2001 .{ }^{29}$ Subjects were randomly assigned to annual PSA testing for 6 years and annual DRE for 4 years. Subjects were referred for biopsy for a PSA level above $4.0 \mathrm{ng} \mathrm{m}{ }^{-1}$ or an abnormal DRE. Detected cancers were treated according to standard practice. Investigators used questionnaires and linkages to the National Death Index to determine cause of death. An endpoint committee reviewed death certificates and adjudicated cause of death.

The screening and control groups were well matched for age, race/ ethnicity and family history of prostate cancer. Subjects were followed for a median of 11.5 years, with vital status ascertained for $98 \%$ of subjects through 7 years of follow-up and for 67\% through 10 years. The screening group was very compliant with PSA testing (85\%) and DRE $(86 \%)$. Screening was associated with a significant increase in cancer detection. After 7 years of follow-up, 2820 cancers were diagnosed in the screening group compared to 2322 in the control group (rate ratio: 1.22, 95\% CI: 1.16-1.29). Tumor characteristics (Gleason score and stage) were more favorable in the screening group, but the study failed to show any mortality benefit. The intervention group actually had more deaths than the control group, though the difference, based on fewer than 100 deaths, was not statistically significant (rate ratio: 1.13 , 95\% CI: 0.75-1.70).
Although the PLCO study did not find a survival benefit with PSA screening, this was not a conclusive outcome because there were a number of important factors that likely biased results towards the null. Because PSA testing was so widespread in the United States at the time the study was launched, nearly $40 \%$ of subjects had undergone at least one PSA test in the previous 3 years. Prostate cancer detection rates decrease with serial PSA testing and there is a shift towards earlier stage, less aggressive cancers, ${ }^{34}$ thus making it more difficult to demonstrate a survival benefit with less than 10 years of follow-up. Additionally, contamination in the control group was substantial with $52 \%$ of subjects in the control group reported PSA testing in the sixth year of the study and $41 \%-46 \%$ also underwent DRE during the study period. Essentially, the study became a comparison of frequent screening versus less frequent screening. Finally, substantial proportions of men with abnormal PSA tests did not undergo a prostate biopsy ${ }^{34}$ and a number of men with early-stage prostate cancers did not undergo attempted curative therapy. ${ }^{29}$

\section{IMPLICATIONS OF SCREENING TRIALS}

After nearly two decades of aggressive PSA screening, particularly in developed countries, the results of the randomized trials and epidemiological data suggest that the absolute survival benefit is small. The tradeoff is that screening will substantially increase the chance of being diagnosed with prostate cancer. ${ }^{35}$ Modeling studies suggest that a substantial proportion of these screening-detected cancers are overdiagnoses, ${ }^{20}$ implying that they would not become clinically important during a man's lifetime. However, because we do not have tumor markers that conclusively identify cancers destined to cause problems, most men with screening-detected cancers will undergo attempted curative treatments with surgery or radiation. ${ }^{21,22}$ These treatments can lead to urinary, sexual and bowel complications that can adversely affect quality of life. ${ }^{23}$ The ERSPC and PLCO have collected quality of life data, but the results have yet to be reported.

The risks of overdiagnosis and overtreatment are major challenges to efforts to reduce the burden of suffering from prostate cancer. Having better tumor markers that are more specific for high-risk cancers would reduce overdiagnosis and ideally reduce unnecessary biopsies. A tumor marker that can provide more accurate prognostic information will help better identify patients most likely to benefit from aggressive treatment.

Experts have also suggested that the focus of cancer control should perhaps shift towards chemoprevention. ${ }^{36}$ Randomized trials of the 5-alpha-reductase inhibitors finasteride and dutasteride have shown that these agents reduce the risk for being diagnosed with prostate cancer by about $25 \% .{ }^{37,38} \mathrm{~A}$ recent guideline from the American Society of Clinical Oncology/American Urological Association has encouraged physicians to discuss chemoprevention with men being screened or considering screening for prostate cancer. ${ }^{39}$ However, the 5-alpha-reductase inhibitors were associated with an increased risk for high-grade cancers (Gleason scores 7-10). Although post hoc analyses suggested that selection and sampling biases might have spuriously elevated the risk, ${ }^{40}$ the guideline acknowledged that these agents still could be inducing aggressive cancers. Given that studies did not have sufficient sample size or follow-up duration to assess prostate cancer mortality, there is no certainty that chemoprevention is beneficial. Indeed, the US Food and Drug Administration recently rejected a request to allow drug manufacturers to market 5-alpha-reductase inhibitors for cancer prevention. ${ }^{41}$

Another promising strategy for minimizing the risk of overtreatment arising from PSA testing is the approach of active surveillance. ${ }^{42}$ 
Instead of immediately treating men with disease at low risk for progression (defined by a PSA level $\leqslant 10 \mathrm{ng} \mathrm{ml}^{-1}$, Gleason score of 6 or less, and a clinical stage T1c or T2a), clinicians monitor the cancer with PSA tests and digital rectal examination every 3-6 months and perform prostate biopsies every 12-24 months. ${ }^{43}$ For men opting for active surveillance, the choice to undergo deferred treatment remains available and can be based on evidence of disease progression (rising PSA, increasing Gleason grade and abnormal DRE) and/or personal preferences.

Observational studies suggest that active surveillance is a reasonable strategy for men with low-risk localized disease. Klotz summarized the experience of 2168 active surveillance patients followed in six observational studies. ${ }^{44}$ Their disease-specific survival was $99.7 \%$ after a median follow-up of 43 months and only about one-third of patients eventually received definitive treatment. An ongoing randomized trial comparing active surveillance versus active treatment among low-risk patients will provide even more information about the safety and acceptability of active surveillance. ${ }^{44}$

In the absence of better tumor markers and clearly effective chemoprevention agents, physicians will need to address the issue of screening with PSA. The recognition that screening involves important tradeoffs has led many professional organizations and guideline developers throughout the world to strongly encourage informed decisionmaking. ${ }^{45-48}$ This implies educating men about the natural history of prostate cancer, the limitations of screening tests, and the risks and benefits of undergoing treatment for early-stage prostate cancer. The goal is to help men make decisions that are concordant with their values. One way to facilitate informed decision-making is to provide patients with decision aids, which can be written, video or webbased. ${ }^{49}$ These are interventions designed to help individuals make specific and deliberative choices among options by providing information about disease, relevant options and outcomes, helping patients clarify their values, and providing guidance for the decision-making process. Decision aids are particularly helpful for issues such as prostate cancer screening where evidence is uncertain and decisions are very sensitive to patient preferences. ${ }^{50}$ A metaanalysis has shown that prostate cancer screening decision aids will increase knowledge and reduce uncertainty about making decisions. ${ }^{51}$ Additionally, after receiving a prostate cancer screening decision aid, subjects were significantly less likely to express interest in undergoing screening or aggressive treatment.

\section{COMPETING FINANCIAL INTERESTS}

The authors declare no competing financial interests.

\section{ACKNOWLEDGMENTS}

Dr Hoffman is supported by the New Mexico VA Health Care System, Albuquerque, NM, USA. The views expressed in this article are those of $\mathrm{Dr}$ Hoffman and not necessarily the Department of Veterans Affairs.

1 Reed AB, Parekh DJ. Biomarkers for prostate cancer detection. Expert Rev Anticancer Ther 2010; 10: 103-14.

2 Catalona WJ, Richie JP, Ahmann FR, Hudson MA, Scardino PT et al. Comparison of digital rectal examination and serum prostate specific antigen in the early detection of prostate cancer: results of a multicenter clinical trial of 6630 men. J Urol 1994; 151: 1283-90.

3 Brawer MK, Chetner MP, Beatie J, Buchner DM, Vessella RL et al. Screening for prostatic carcinoma with prostate specific antigen. J Urol 1993; 147: 841-5.

4 Mettlin C, Jones G, Averette H, Gusberg SB, Murphy GP. Defining and updating the American Cancer Society guidelines for the cancer-related checkup: prostate and endometrial cancers. CA Cancer J Clin 1993; 43: 42-6.
5 American Urological Association. Early detection of prostate cancer and use of transrectal ultrasound. In: American Urological Association 1992 Policy Statement Book. Baltimore, MD: American Urological Association; 1992. p4-20.

6 Sirovich BE, Schwartz LM, Woloshin S. Screening men for prostate and colorectal cancer in the United States: does practice reflect the evidence? JAMA 2003; 289: 1414-20.

7 Silverberg E. Cancer statistics. 1986. CA Cancer J Clin 1986; 36: 9-25.

8 Altekruse SF, Kosary C, Krapcho M, Neyman N, Aminou R et al. SEER Cancer Statistics Review 1975-2007. Bethesda, MD: National Cancer Institute; 2010. http://seer.cancer.gov/csr/1975_2007/ (accessed 12 December 2010).

9 GLOBOCAN 2008. Cancer Fact Sheet. Prostate Cancer Incidence and Mortality Worldwide in 2008. Lyon: International Agency for Research on Cancer; 2008. http://globocan.iarc.fr/factsheets/cancers/prostate.asp (accessed 12 December 2010).

10 Stanford JL, Stephenson RA, Coyle LM, Cerhan J, Correa R et al. Prostate cancer trends 1973-1995. Bethesa, MD: SEER Program, National Cancer Institute; 1999.

11 Feuer EJ, Merrill RM, Hankey BF. Cancer surveillance series: interpreting trends in prostate cancer-Part II: Cause of death misclassification and the recent rise and fall in prostate cancer mortality. J Nat/ Cancer Inst 1999; 91: 1025-32.

12 Etzioni R, Tsodikov A, Mariotto A, Szabo A, Falcon S et al. Quantifying the role of PSA screening in the US prostate cancer mortality decline. Cancer Causes Control 2008; 19: 175-81.

13 Schroder $\mathrm{FH}$, Bangma $\mathrm{CH}$. The European Randomized Study of Screening for Prostate Cancer (ERSPC). Br J Urol 1997; 79 (Suppl 1): 68-71.

14 Gohagan JK, Prorok PC, Hayes RB, Kramer BS. The Prostate, Lung, Colorectal and Ovarian (PLCO) Cancer Screening Trial of the National Cancer Institute: history, organization, and status. Control Clin Trials 2000; 21: 251S-72S.

15 Labrie F, Candas B, Dupont A, Cusan L, Gomez JL et al. Screening decreases prostate cancer death: first analysis of the 1988 Quebec prospective randomized controlled trial. Prostate 1999; 38: 83-91.

16 Finne P, Fallah M, Hakama M, Ciatto S, Hogosson J et al. Lead-time in the European Randomised Study of Screening for Prostate Cancer. Eur J Cancer 2010; 46: 3102-8.

17 Draisma G, Boer R, Otto SJ, van der Cruijsen IW, Damhuis RA et al. Lead times and overdetection due to prostate-specific antigen screening: estimates from the European Randomized Study of Screening for Prostate Cancer. J Natl Cancer Inst 2003; 95: 868-78.

18 Bill-Axelson A, Holmberg L, Filen F, Ruutu M, Garmo H et al. Radical prostatectomy versus watchful waiting in localized prostate cancer: the Scandinavian Prostate Cancer Group-4 Randomized Trial. J Natl Cancer Inst 2008; 100: 1144-54.

19 Ilic D, O'Connor D, Green S, Wilt T. Screening for prostate cancer: a Cochrane systematic review. Cancer Causes Control 2007; 18: 279-85.

20 Draisma G, Etzioni R, Tsodikov A, Mariotto A, Wever E et al. Lead time and overdiagnosis in prostate-specific antigen screening: importance of methods and context. J Natl Cancer Inst 2009; 101: 374-83.

21 Bubolz T, Wasson JH, Lu-Yao G, Barry MJ. Treatments for prostate cancer in older men: 1984-1997. Urology 2001; 58: 977-82.

22 Cooperberg MR, Broering JM, Latini DM, Litwin MS, Wallace KL et al. Patterns of practice in the United States: insights from CaPSURE on prostate cancer management. Curr Urol Rep 2004; 5: 166-72.

23 Wilt TJ, MacDonald R, Rutks I, Shamliyan TA, Taylor BC et al. Systematic review: comparative effectiveness and harms of treatments for clinically localized prostate cancer. Ann Intern Med 2008; 148: 435-48.

24 Lu-Yao GL, Albertsen P, Warren J, Yao SL. Effect of age and surgical approach on complications and short-term mortality after radical prostatectomy-a populationbased study. Urology 1999; 54: 301-7.

25 U.S. Preventive Services Task Force.Screening for prostate cancer: recommendation and rationale. Ann Intern Med 2002; 137: 915-6.

26 von Eschenbach A, Ho R, Murphy GP, Cunningham M, Lins N. American Cancer Society guideline for the early detection of prostate cancer: update 1997. CA Cancer J Clin 1997; 47: 261-4.

27 Prostate-specific antigen (PSA) best practice policy. American Urological Association (AUA). Oncology (Williston Park) 2000; 14: 267-72, 77-8, 80 passim.

28 Schroder FH, Hugosson J, Roobol MJ, Tammela TL, Ciatto S et al. Screening and prostate-cancer mortality in a randomized European study. N Engl J Med 2009; 360: 1320-8.

29 Andriole GL, Crawford ED, Grubb RL 3rd, Buys SS, Chia D et al. Mortality results from a randomized prostate-cancer screening trial. N Engl J Med 2009; 360: 1310-9.

30 Greene KL, Albertsen PC, Babaian RJ, Carter HB, Gann PH et al. Prostate specific antigen best practice statement: 2009 update. J Urol 2009; 182: 2232-41.

31 Roobol MJ, Kerkhof M, Schröder FH, Cuzick J, Sasieni P et al. Prostate cancer mortality reduction by prostate-specific antigen-based screening adjusted for nonattendance and contamination in the European Randomised Study of Screening for Prostate Cancer (ERSPC). Eur Urol 2009; 56: 584-91.

32 Kerkhof M, Roobol MJ, Cuzick J, Sasieni P, Roemeling S et al. Effect of the correction for noncompliance and contamination on the estimated reduction of metastatic prostate cancer within a randomized screening trial (ERSPC section Rotterdam). Int J Cancer 2010; 127: 2639-44.

33 Hugosson J, Carlsson S, Aus G, Bergdahl S, Khatami A et al. Mortality results from the Goteborg randomised population-based prostate-cancer screening trial. Lancet Oncol 2010; 11: 725-32.

34 Grubb RL 3rd, Pinsky PF, Greenlee RT, Izmirlian G, Miller AB et al. Prostate cancer screening in the Prostate, Lung, Colorectal and Ovarian Cancer Screening Trial: update on findings from the initial four rounds of screening in a randomized trial. BJU Int 2008; 102: 1524-30. 
35 Brawley OW, Ankerst DP, Thompson IM. Screening for prostate cancer. CA Cancer J Clin 2009; 59: 264-73.

36 Esserman L, Shieh Y, Thompson I. Rethinking screening for breast cancer and prostate cancer. JAMA 2009; 302: 1685-92.

37 Thompson IM, Goodman PJ, Tangen CM, Lucia MS, Miller GJ et al. The influence of finasteride on the development of prostate cancer. N Engl J Med 2003; 349: 215 24.

38 Andriole GL, Bostwick DG, Brawley OW, Gomella LG, Marberger M et al. Effect of dutasteride on the risk of prostate cancer. N Engl J Med 2010; 362: 1192-202.

39 Kramer BS, Hagerty KL, Justman S, Somerfield MR, Albertsen PC et al. Use of 5-alpha-reductase inhibitors for prostate cancer chemoprevention: American Society of Clinical Oncology/American Urological Association 2008 Clinical Practice Guideline. J Clin Oncol 2009; 27: 1502-16.

40 Thompson IM, Tangen CM, Goodman PJ, Lucia MS, Klein EA. Chemoprevention of prostate cancer. J Urol 2009; 182: 499-507.

41 Kolota G. FDA Panel rejects expanded use of prostate drug. Health Section. New York Times, 1 December 2010.

42 Dall'Era MA, Carroll PR. Outcomes and follow-up strategies for patients on active surveillance. Curr Opin Urol 2009; 19: 258-62.

43 Thompson I, Thrasher JB, Aus G, Burnett AL, Canby-Hagino ED et al. Guideline for the management of clinically localized prostate cancer: 2007 update. J Urol 2007; 177: 2106-31.
44 Klotz L, Zhang L, Lam A, Nam R, Mamedov A et al. Clinical results of long-term followup of a large, active surveillance cohort with localized prostate cancer. $J$ Clin Oncol 2010; 28: 126-31

45 Wolf AM, Wender RC, Etzioni RB, Thompson IM, D'Amico AV et al. American Cancer Society guideline for the early detection of prostate cancer: update 2010. CA Cancer J Clin 2010; 60: 70-98.

46 U.S. Preventive Services Task Force. Screening for prostate cancer: U.S.Preventive Services Task Force recommendation statement. Ann Intern Med 2008; 149: 185 91.

47 Cancer Council Australia. Position statement. Prostate cancer screening. Sydney, NSW: Australian Health Ministers' Advisory Council; 2010. http://www.cancer.org.au/ Healthprofessionals/PositionStatements/prostatecancer.htm (accessed 13 December 2010).

48 Prostate Cancer Risk Management Programme. London: NHS Cancer Screening Press Office; 2010. http://www.cancerscreening.nhs.uk/prostate/index.html (accessed 13 December 2010).

49 Barry MJ. Health decision aids to facilitate shared decision making in office practice. Ann Intern Med 2002; 136: 127-35.

50 Rimer BK, Briss PA, Zeller PK, Chan EC, Woolf SH. Informed decision making: what is its role in cancer screening? Cancer 2004; 101: 1214-28.

51 Volk RJ, Hawley ST, Kneuper S, Holden EW, Stroud LA et al. Trials of decision aids for prostate cancer screening: a systematic review. Am J Prev Med 2007; 33: 428-34. 